\title{
QPACE - a QCD parallel computer based on Cell processors
}

H. Baier ${ }^{1}$, H. Boettiger ${ }^{1}$, M. Drochner ${ }^{2}$, N. Eicker ${ }^{2,3}$, U. Fischer ${ }^{1}$, Z. Fodor ${ }^{3}$, A. Frommer ${ }^{3}$, C. Gomez ${ }^{4}$, G. Goldrian ${ }^{1}$, S. Heybrock ${ }^{5}$, D. Hierl ${ }^{5}$, M. Hüsken ${ }^{3}$, T. Huth ${ }^{1}$, B. Krill ${ }^{1}$, J. Lauritsen ${ }^{1}$, T. Lippert ${ }^{2,3}$, T. Maurer ${ }^{* 5}$, N. Meyer ${ }^{5}$, A. Nobile ${ }^{5}$, I. Ouda ${ }^{6}$, M. Pivanti ${ }^{7}$, D. Pleiter ${ }^{\dagger 8}$, A. Schäfer ${ }^{5}$, H. Schick ${ }^{1}$, F. Schifano ${ }^{7}$, H. Simma ${ }^{8,9}$, S. Solbrig ${ }^{5}$, T. Streuer ${ }^{5}$, K.-H. Sulanke ${ }^{8}$, R. Tripiccione ${ }^{7}$, J.-S. Vogt $^{1}$, T. Wettig ${ }^{5}$, F. Winter ${ }^{8}$

${ }^{1}$ IBM Deutschland Research \& Development GmbH, 71032 Böblingen, Germany

${ }^{2}$ Research Center Jülich, 52425 Jülich, Germany

${ }^{3}$ University of Wuppertal, 42119 Wuppertal, Germany

${ }^{4}$ IBM La Gaude, Le Plan du Bois, La Gaude 06610, France

${ }^{5}$ Department of Physics, University of Regensburg, 93040 Regensburg, Germany

${ }^{6}$ IBM Rochester, 3605 HWY 52 N, Rochester MN 55901-1407, USA

${ }^{7}$ University of Ferrara, 44100 Ferrara, Italy

${ }^{8}$ Deutsches Elektronen Synchrotron (DESY), 15738 Zeuthen, Germany

${ }^{9}$ Department of Physics, University of Milano-Bicocca, 20126 Milano, Italy

QPACE is a novel parallel computer which has been developed to be primarily used for lattice QCD simulations. The compute power is provided by the IBM PowerXCell 8i processor, an enhanced version of the Cell processor that is used in the Playstation 3. The QPACE nodes are interconnected by a custom, application optimized 3-dimensional torus network implemented on an FPGA. To achieve the very high packaging density of 26 TFlops per rack a new water cooling concept has been developed and successfully realized. In this paper we give an overview of the architecture and highlight some important technical details of the system. Furthermore, we provide initial performance results and report on the installation of 8 QPACE racks providing an aggregate peak performance of 200 TFlops.

The XXVII International Symposium on Lattice Field Theory - LAT2009

July 26-31 2009

Peking University, Beijing, China

\footnotetext{
*Speaker.
}

† Speaker. 


\section{Introduction}

In the past custom-designed supercomputers have contributed a significant fraction of the compute cycles available for lattice QCD calculations. They did not only make compute power available at affordable costs, they also ensured the availability of highly scalable architectures needed for many calculations. However, design, production and deployment of such cost-efficient machines becomes more challenging with prices per GFlops for commodity systems going down and technology becoming more complex. For example, the latest generation of custom machines, apeNEXT [1] and QCDOC [2], was based on custom designed processors. Given the costs and the risks involved in an ASIC design, this has become less of an option today.

In the QPACE project we therefore decided to explore a different concept. Instead of a custom designed processor we select fast commodity processors which we interconnect via a custom network. This network is implemented through a companion processor which we call the network processor (NWP) and for which we use a re-configurable Field Programmable Gate Array (FPGA) instead of an ASIC. The nodes consisting of a commodity processor and a network processor then have to be integrated into a cost-efficient system design.

To realize such a concept a suitable commodity processor has to be selected. Achieving good performance for key lattice QCD kernels is an obvious criterion. Using a powerful multi-core processor helps to reduce the required number of nodes (which is likely to reduce costs) and relax the requirements for the network bandwidth. One also has to take into account that connecting the NWP to the I/O interface of a complex processor is a technical challenge. At the time of starting the project we identified the IBM PowerXCell $8 \mathrm{i}$ processor as a suitable candidate.

For lattice QCD applications to scale on a large number of processors it is not only the network bandwidth that matters. For typically used algorithms, data are communicated using relatively small messages, and the network latency will thus have a major impact on the efficiency that can be achieved. Frequently used custom network technologies (e.g., Infiniband) provide a bandwidth which exceeds our requirements, which for the given processor we estimated to be $O(1)$ GBytes/sec per node and direction. Latencies, however, are typically more than $O(10) \mu$ sec. On the PowerXCell $8 \mathrm{i}$ running at $3.2 \mathrm{GHz}$ this corresponds to $O(30,000)$ clock cycles. Using custom-designed hardware and software our goal was to bring this down to $O(1) \mu$ sec.

A final challenge in terms of system design is the cost-efficient integration of the basic building blocks into a system. While development and procurement costs still constitute the largest fraction of the total cost of ownership, operational costs, in particular costs for electricity and cooling, are becoming more and more relevant. Indeed, power-efficient computing has become a major topic in the area of HPC.

After having outlined the requirements and challenges of the QPACE project we will in the next section, section 2, discuss the processor in more detail. In the following sections 3 and 4 we will provide an overview of the architecture and highlight some of the hardware components designed in this project. In section 5 we will focus on one of the core components, the network processor, and its implementation on an FPGA. Section 6 describes the concept of the torus network and provides results from some initial performance measurements. We then continue by highlighting the novel cooling system and the power efficiency of the system in section 7. Section 8 explains how the system is integrated with a front-end system. Section 9 provides an overview of initial ex- 
periences implementing application programs for QPACE. Before presenting our conclusions in section 11 we briefly describe the organization of this project in section 10 .

\section{PowerXCell 8i processor}

The PowerXCell 8i processor is a more recent implementation of the Cell Broadband Engine Architecture [3]. A first implementation has been developed by Sony, Toshiba and IBM with the first major commercial application being Sony's PlayStation 3. The PowerXCell 8i is an enhanced version of that processor with support for high-performance double precision operations, IEEEcompliant rounding, and a DDR2 memory interface.

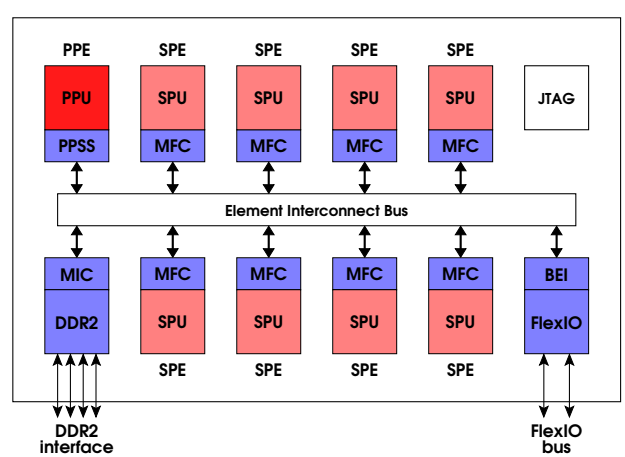

(a) PowerXCell 8i processor

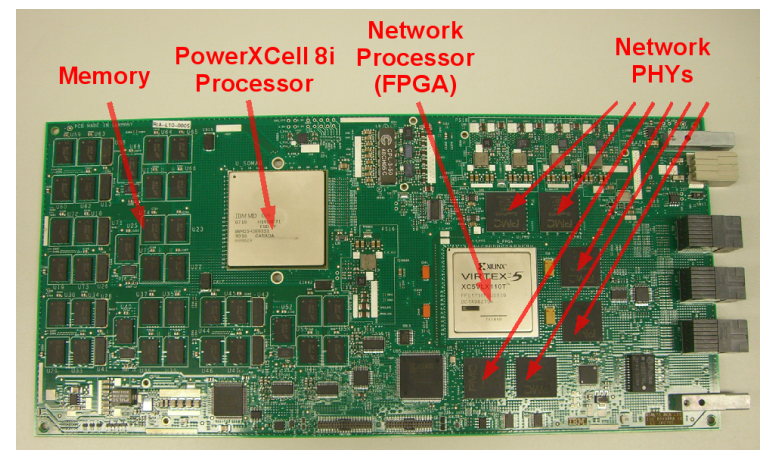

(b) QPACE Node card

Figure 1: The left panel shows a schematic overview of the PowerXCell 8i processor with 8 Synergistic Processing Elements (SPE) and a Power Processing Element (PPE). The other components are described in the text. The right panel shows the QPACE node card.

The PowerXCell 8i comprises multiple cores (see Fig. 1a) including the Power Processing Element (PPE), which is a standard PowerPC core that can, e.g., be used for running the operating system Linux. From there threads can be started on the 8 Synergistic Processing Elements (SPE). These cores as well as the Memory Interface Controller (MIC) and the Broadband Engine Interface (BEI) are interconnected via the Element Interconnect Bus (EIB). This bus has a very high bandwidth of up to 200 GBytes/sec. The JTAG interface provides access to processor registers for debugging.

The real compute power is provided by the 8 SPEs. Each of them consists of a Synergistic Processing Unit (SPU) and a Memory Flow Controller (MFC). In each clock cycle the SPU can perform a multiply-add operation on a vector of 4 (2) single-precision (double-precision) floatingpoint numbers. This gives a peak floating-point performance per SPU of 25.6 or 12.8 GFlops in single or double precision, respectively. With a peak performance of 205 (102) single-precision (double-precision) GFlops the PowerXCell 8i was the most powerful commercially available processor at the start of the QPACE project. The same processor is also used in the Roadrunner supercomputer [4], which at the time of writing this paper is still number one on the Top500 list [5].

The memory hierarchy of the processor is non-trivial. Each of the SPUs has its own Local Store (LS), a 256 kBytes on-chip memory from which up to one 16 Byte word per clock cycle can 
be loaded or stored to or from the SPU's register file. Loading and storing data from and to other devices attached to the EIB is handled by the Direct Memory Access (DMA) engine in the MFC. The interface to the external memory, the MIC, provides a bandwidth of 25.6 GBytes/sec. This interface is shared by all SPEs, thus reducing the bandwidth per clock cycle and SPE to 1 Byte peak.

From a naive comparison with the bandwidth requirements of the most important application kernel, the fermion matrix-vector multiplication, this bandwidth is small compared to the floating-point performance. Let us consider the case of Wilson-Dirac fermions, where the ratio of single-precision Flops per Byte which has to be loaded or stored is 0.9. Comparing the SPU single-precision performance and the bandwidth between register file and LS we have a ratio of 8 Flops/16 Bytes, i.e., the bandwidth exceeds our requirements. But for loads from and stores to main memory this ratio changes to 64 Flops/8 Bytes. This simple comparison of hardware characteristics and one of the important application performance signatures indicates that it is possible to achieve a good sustained performance if implementation is heavily optimized with respect to reducing memory access and keeping data in the LS. For a more detailed performance analysis see $[6,7,8]$.

\section{Architecture overview}

The smallest building block of QPACE is the node card. Node cards are inserted into backplanes, each of which can host up to 32 node cards plus 2 root cards. The latter can be used to monitor and control up to 16 node cards mounted in the same row. One QPACE rack can house up to 8 backplanes, i.e., the maximum number of node cards per rack is 256 . In Fig. 2a only 4 units with 32 node cards each are visible. Due to the water-cooling sub-system there is no need for air being able to flow from one side of the rack to the other. Therefore the other side of the rack can be used to mount another 128 node cards.

Power is distributed via the backplanes to node and root cards. Three hot-swappable power supply units (PSU) are attached to each backplane, one of them being redundant. To connect the backplane and the PSU we designed a PSU interface board which makes it possible to access the PSUs via a serial I2C link. These links connect all PSUs within one rack to a superroot card which is the central PSU monitoring and control instance.

The QPACE architecture comprises 3 different types of networks:

- The torus network is a low-latency, high-bandwidth network. This network connects each node card to its 6 nearest neighbors within a torus. We will describe this network in more detail in section 6 .

- The Ethernet network connects node cards as well as the root and superroot cards to the front-end system. It is used for booting and I/O. (See section 8 for more details.)

- The global signal network is a simple 2-wire tree network. It can be used for fast evaluation of global conditions and synchronization of the node cards as well as distribution of an interrupting kill signal to all node cards of a particular partition. 


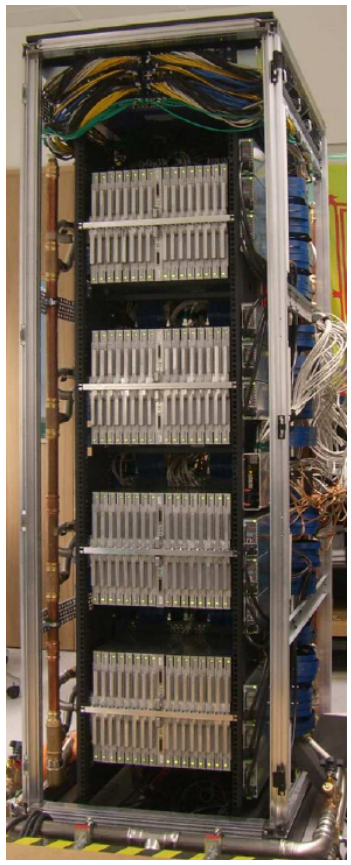

(a) Rack

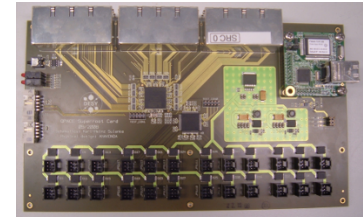

(b) Superroot card

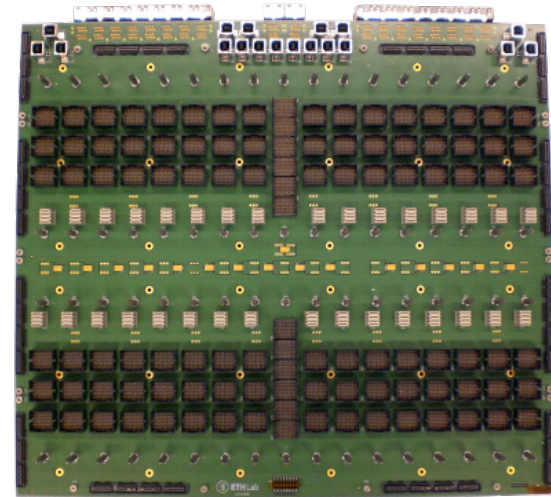

(c) Backplane

Figure 2: The left panel shows a QPACE rack during assembly in the IBM Böblingen lab. Visible are 4 units with 32 node cards each. The vertical copper pipes at the left side are part of the cooling system. Pictures (b) and (c) show a QPACE superroot card and a backplane.

A global clock signal is distributed via the root cards. This clock is used to generate the reference clock for the torus network. The main purpose of this global clock tree is to maximize alignment of the clocks at transmit and receive side.

Also integrated in the rack are 7 Ethernet switches to which all 256 node cards, 16 root cards and the superroot card are connected.

\section{Hardware details}

The QPACE node card is shown in Fig. 1b. The left part of the node card contains the DDR2 memory chips and the PowerXCell 8i. Each node card provides 4 GBytes of main memory. The number of memory modules has been optimized for maximum bandwidth. A small microcontroller is used as a Service Processor (SP). This controller not only monitors and manages the PowerXCell 8i, it also controls the on-board voltages and watches the temperatures. If voltage or temperature thresholds are exceeded the node card is automatically switched off.

The components sitting on the right half of the PCB are special for the QPACE architecture, i.e., the FPGA and a set of devices, called PHYs, which implement the physical layer of the torus network (see section 6 for more details) and the Ethernet interface.

The root card comprises a microcontroller which is operated using an Linux version optimized for embedded systems. It can be accessed from a front-end system via its Ethernet interface. The controller and the service processors of the 16 node cards mounted in the same backplane row are 
attached to a shared RS-485 serial bus, thus proving front-end access to the service processors. The root card furthermore controls the reset lines and 2 other serial links going to each of the node cards. The necessary switching logic has been implemented on a Complex Programmable Logic Devices (CPLD). A second CPLD is used to implement the second level of the global signal tree network.

The higher levels of the global signal tree network are implemented on the superroot cards (see Fig. 2b) again using a CPLD. Here a second CPLD is used to implement the logic for the links to the PSUs. Via these links the PSUs can be monitored and controlled. To access both devices an Ethernet to serial link converter is used.

Finally, the last board designed for QPACE is the backplane, a fully passive board. Its main tasks are power distribution and routing of a large number of signals (mostly high-speed signals) connecting the node cards among each other, or node card and root card as well as node cards and cable connectors. As a result of the compactness of the design a relatively dense placement of the connectors was required. In particular, at the boundary of the board almost all available space is occupied by cable connectors (see Fig. 2c).

\section{Network processor}

A core component of the QPACE architecture is the network processor (NWP). This device acts as an I/O fabric similar to the chips which on more standard compute boards connect the processor to external peripheral devices. While in commodity products these chips are implemented on an ASIC, we use a Field Programmable Gate Arrays (FPGA), which is a rather unique feature of the QPACE node card.

FPGAs are also semiconductor devices, but they are re-programmable, i.e., the functional behavior of the device can be changed at any time. This means that development risks are significantly reduced as the logic can be modified even after deployment of the hardware. The disadvantage of using FPGAs is that they are typically slower than custom ASICs and the price per device is typically higher. ${ }^{1}$

An FPGA consists of a large number of programmable logic blocks. A hierarchy of reconfigurable interconnects allows to glue these blocks together in different ways. In some FPGAs so-called hard cores are embedded which provide a fast implementation of a certain functionality. For instance, in the QPACE NWP an Ethernet Medium Access Control (MAC) core is used to implement the lower part of the data link layer of the Gigabit Ethernet interface.

To define the functional behavior of an FPGA the developer implements a design using a hardware description language (usually VHDL). Using a complex tool chain this design is translated into a so-called bitstream which is used to program the FPGA after power-on of the node card.

The FPGA which we chose for the QPACE project is the Virtex-5 LX110T by Xilinx. This FPGA is of medium size (and therefore affordable in price) with an amount of logic blocks which we estimated to be sufficient for our design. Furthermore, the number of available general purpose I/O pins and fast transceivers was large enough to connect the FPGA to the PowerXCell 8i, the network PHYs and some other slower devices. As performance was expected to become a challenge we selected the highest available speed grade for this device.

\footnotetext{
${ }^{1}$ For small projects this disadvantage is typically more than compensated for by the reduction in development costs.
} 
In Fig. 3 we give an overview of the internal structure of our NWP design. The entities depicted in its upper part contain IBM's implementation of the physical and data link layer towards the PowerXCell $8 \mathrm{i}$ as well as the interface to the application logic designed by the academic partners. This application logic can be categorized in the following way:

- Torus network block including 6 links (described in more detail in section 6).

- Gigabit Ethernet interface.

- Various devices connected to slow interfaces, e.g.,

- 2 serial links connecting the PowerXCell 8i to the service processor and the root card.

- An interface to the global signal tree network.

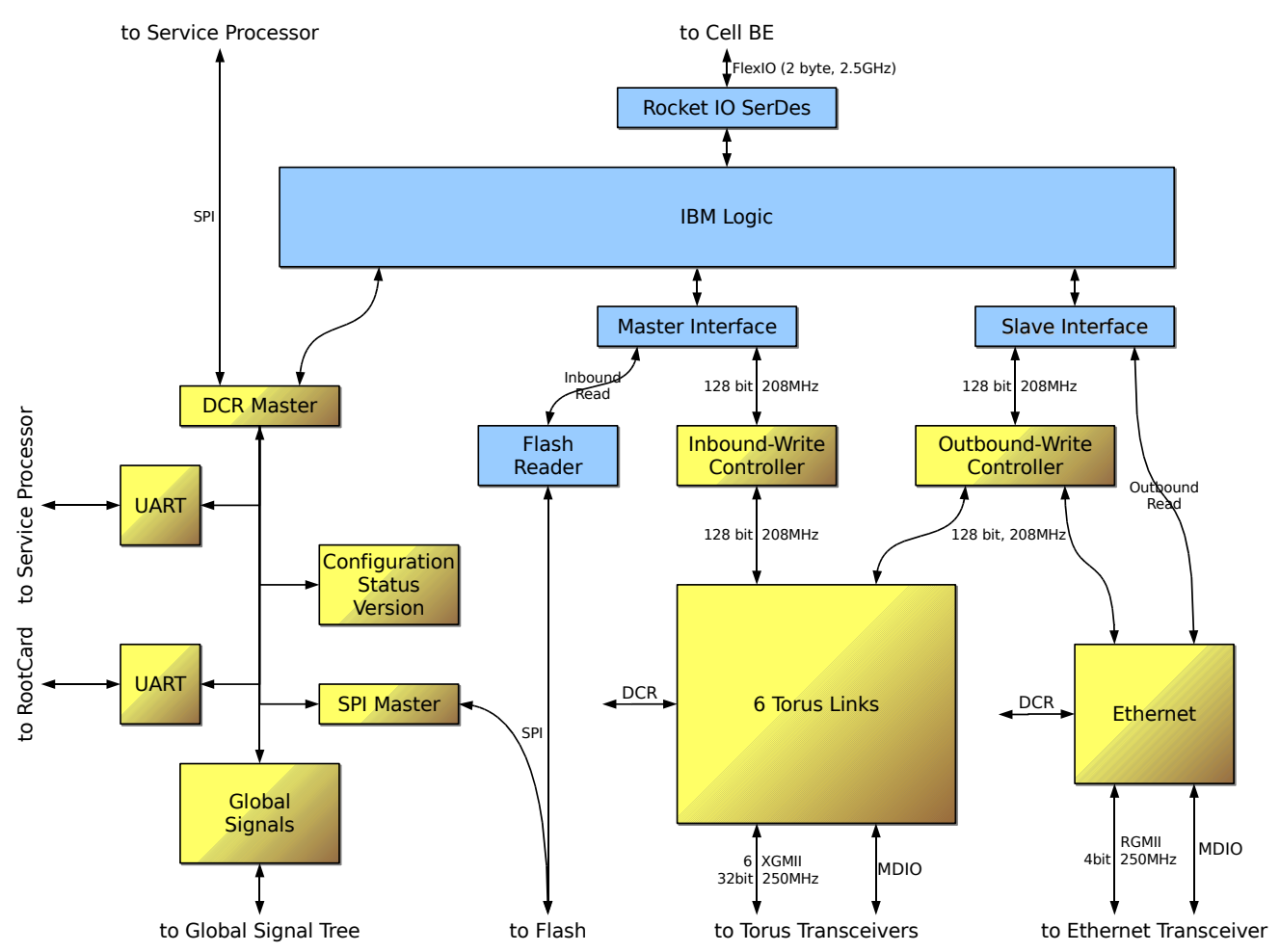

Figure 3: Overview of the internal architecture of the Network Processor (NWP). The blue boxes refer to entities implemented by IBM, the others have been implemented by the academic partners.

With the NWP basically being a large I/O fabric, most entities are connected to external lines. This includes:

- Two 8-bit wide (full-duplex) bi-directional high-speed links connecting the NWP to the PowerXCell 8i with a bandwidth of up to 5 GBytes/sec per direction.

- Six (full-duplex) bi-directional links to the 10-Gigabit Medium Independent Interfaces (XGMII) of the torus network PHYs. Per link and clock cycle 32 bits can be sent and received at a clock speed of $250 \mathrm{MHz}$. 
- One common Reduced Gigabit Medium Independent Interface (RGMII) interface connecting the NWP to an external Ethernet 1000BASE-T physical transceiver. Running at $250 \mathrm{MHz}$ per direction 4 bits per cycle can be communicated.

- A 4-bit interface (2 differential lines per direction) interface to the global signal tree network.

- Lines connected to the 2 Universal Asynchronous Receiver Transmitters (UART) which are interfaces to the serial links.

Major challenges had to be solved to connect the PowerXCell 8i and the NWP via a high-speed link. The link consists of 2 links with 8 lines per direction each. The transceiver technology used in the processor is called FlexIO, a technology from Rambus which allows for a speed-up of data rates up to $8 \mathrm{GHz}$. On the other side these lines are connected to Xilinx RocketIO transceivers which are able to sustain data rates up to $3.75 \mathrm{Gbit} / \mathrm{sec}$. With both technologies not being fully compatible careful tuning of the design at the physical link layer was required [9]. We have verified that the link can be operated in a stable manner at $2.5 \mathrm{GHz}$ without bit errors during several hours on hundreds of node cards. At this speed the link has a bandwidth of 5 GBytes/sec per direction, which roughly balances the bandwidth of all 6 torus network links. Single node cards have been successfully tested at $3 \mathrm{GHz}$.

While the high-speed network interfaces are connected via a fast data path to the processorNWP link, the slow interfaces are accessed via a simple shared Device Control Register (DCR) bus. This bus is also used to access the configuration and status registers of the network interfaces.

In Tab. 1a we show for some of the most relevant logic blocks, like Flip Flops, Lookup Tables (LUT) and pins, as well as some special features, like block memory modules (BRAM), how many of them are actually used to fit our design into the given FPGA. As can be seen from this table the filling fraction of the FPGA is very high. As a consequence mapping the design taking all relevant timing constraints into account has become a challenge. We were therefore forced to compromise on the clock speed. Currently a core part of the design is operated at $166 \mathrm{MHz}$, which is still significantly below our target speed of $208.3 \mathrm{MHz}$.

To analyze the FPGA's resource utilization in more detail, we have split the number of used registers and LUTs according to the design entities. The results are shown in Tab. 1b. About 50\% of these resources are occupied by the logic which implements the interface to the PowerXCell $8 \mathrm{i}$. The next-to-biggest consumer is the torus network with about $6 \%$ per link.

\section{Torus network}

The torus network has been designed to meet the following requirements:

- Provide connectivity with the six nearest neighbors within a torus topology.

- The network should allow to send and receive messages directly from the SPE of one node to an SPE of a neighboring node without support from the PPE and without copying the data to main memory. 


\begin{tabular}{|l|r|r|r|}
\hline Primitive & Available & Used & Usage \\
\hline Slices & 17,280 & 16,029 & $92 \%$ \\
LUT-Flip Flop pairs & 69,120 & 51,018 & $73 \%$ \\
\hline Slice registers & 69,120 & 38,212 & $55 \%$ \\
Slice LUTs & 69,120 & 36,939 & $53 \%$ \\
BRAM/FIFO & 148 & 53 & $35 \%$ \\
\hline Pins & 680 & 656 & $96 \%$ \\
\hline
\end{tabular}

(a) Primitives utilization

\begin{tabular}{|l|r|r|r|r|}
\hline & \multicolumn{2}{|c|}{ Registers } & \multicolumn{2}{c|}{ LUTs } \\
\hline PowerXCell 8i interface & 20,225 & $53 \%$ & 16,915 & $46 \%$ \\
Torus network & 13,672 & $36 \%$ & 14,252 & $39 \%$ \\
Ethernet & 1,537 & $4 \%$ & 894 & $2 \%$ \\
Other & 2,778 & $7 \%$ & 4,878 & $13 \%$ \\
\hline
\end{tabular}

(b) Usage per design entity

Table 1: FPGA device utilization: The upper table compares the utilization of the available logic blocks and specific features for our current design. In the lower table we split the number of used blocks and features according to the different design entities.

- The bandwidth performance goal was set to $O(1)$ GByte/s per link and direction. From our performance estimates $[6,7]$ we expected this to be sufficiently large such that the network bandwidth would not become a bottleneck for our applications.

- We aimed for a small latency for LS to LS copy operations of the order of $O(1) \mu$ sec. This latency is small compared to typical commodity solutions but larger than for previous custom processors where the networking functionality had been integrated on the chip (so-called system-on-a-chip designs).

To meet these requirements and to ensure that the logic of all 6 links would fit in our FPGA required an optimized design.

In order to minimize protocol overhead and to avoid handshake between transmitter and receiver we adopted a two-sided communication model. When node $A$ initiates transmission of a message to node $B$ it has to rely on node $B$ initiating a corresponding receive operation. It is the responsibility of the programmer to ensure that the communication parameters (e.g., message size) and the order of the communication instructions match.

As a further simplification we restrict message sizes to be a multiple of 128 Bytes as well as source and destination addresses to be 128 Byte aligned. This is a natural choice for the PowerXCell $8 \mathrm{i}$ as for the internal bus, the EIB, a similar simplification had been made. On the torus network link the messages are split in packets which consist of a 4 Byte header, a 128 Byte payload and a 4 Byte checksum. This means that the protocol overhead added at the data link layer increases the number of transmitted bytes by only $6 \%$. 


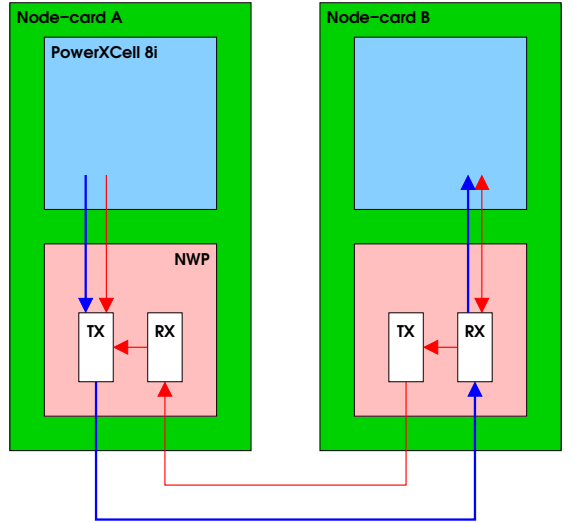

(a) Data and control paths

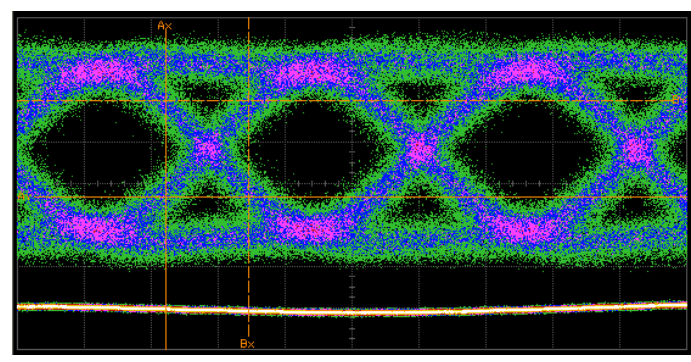

(b) Eye diagram

Figure 4: The left panel shows the data (blue) and control (red) paths for the torus network. The right figure shows an eye diagram measured close to a torus network receiver. For this measurement a link running at $3.125 \mathrm{GHz}$ was selected which is routed through about $40 \mathrm{~cm}$ of PCB, $50 \mathrm{~cm}$ cable, 2 PCB-PCB connectors and 2 PCB-cable connectors.

The communication of messages between different processors is split into multiple transactions. In our design for all transactions data is pushed by the source to the destination. For each transaction we have to ensure that no loss of data occurs. We do this by employing different mechanisms:

1. If the source device for a particular transaction initiates a push operation the write request may not be acknowledged once no more buffer space is available in the destination device, i.e. any further write operation is blocked.

2. The source device sends the data but keeps a copy of each packet until receipt of the packet has been acknowledged by the receiver. If receipt is not acknowledged, it automatically retransmits the packet.

3. Finally, the source device may be requested to wait until it is provided with a credit by the destination device. This credit may contain additional information where to write the data.

If any of these transactions is stalled then back-pressure will be generated

Also data integrity must be preserved during all transactions. In our design we make the (usual) assumption that the data buses within a chip are safe, while external links need to be protected by checksums.

Within our two-sided communication model the sender starts a send operation simply by moving a message using a DMA put operation from the attached storage (e.g., the LS) to one of the transmit (TX) FIFOs ${ }^{2}$ in the NWP (see Fig. 4a). There is one such 2 kByte large FIFO for each of the 6 links. Once the data arrive in this FIFO the data are queued for transmission and will automatically be sent out without further intervention of the processor. The necessary control information

\footnotetext{
${ }^{2}$ FIFO stands for First In, First Out and describes a storage entity with a write port and a read port. Data written to this FIFO can only be read in the same order as they were written.
} 
is encoded in the address used in the DMA put operation. When the FIFO is full further writing is disabled, which will eventually create back-pressure between NWP and SPE.

Once the data is queued for sending, it will immediately be transmitted over the network link. Since the receiver may either not be able to accept the data in its receive buffers or identify data corruption the sender must keep a copy of the packet until the receiver has returned a 4 Byte command packet. This packet contains either an ACK (acknowledge) or NACK (not acknowledge). In the latter case the sender will automatically retransmit the packet.

So far, all transactions are initiated by the sender. To initiate the last step, in which data are moved from the NWP of the receiving node to the processor (or eventually main memory), the sender has to provide a credit to the receiving link in the NWP. Once data have arrived in the receive buffer and a matching credit is found the link requests access to the interface towards the PowerXCell 8i. After an arbiter provided a grant, data are moved to their destination and the processor gets notified once the operation has completed.

As a result of the requirement that any of the SPEs can be an endpoint of a communication we have to take into account that multiple pairs of SPEs on neighboring nodes may share the same physical link. We employed the concept of virtual channels. By using different channels up to 8 pairs of sender and receiver are logically separated. In the receiver this requires the instantiation of a second level of arbitration and logic to reorder data packets from different channels according to the available credits.

Although protocol overhead has been minimized, there is quite some flexibility to control the destination address of a network packet. The final destination address is determined as a sum of 3 addresses:

- A base address defined by the receiver. It typically points to a page in main memory or the base address of the LS and is not changed after initialization of the network. One such address can be defined per link and virtual channel.

- A remote offset which is provided by the sender and transmitted in the packet header.

- A local offset defined by the receiver when providing a credit. This local offset, e.g., defines the address of the receive buffer within the LS relative to the base address.

For the physical layer of the link we decided to use 10-Gigabit Ethernet (10-GbE). This layer is implemented by external network devices, called PHYs. Data is moved out of the Network Processor (NWP) in a 32-bit wide data bus at a speed of $250 \mathrm{MHz}$. The PHY serializes and encodes the data. Per link and direction there are 4 serial lines with a data rate of $2.5 \mathrm{GBit} / \mathrm{sec}$ each. ${ }^{3}$ Taking the overhead of 10/8-bit encoding into account, this results in a gross bandwidth of $1 \mathrm{GByte} / \mathrm{sec}$ per link and direction.

Because of the compactness of the system design we could limit the maximum cable length to $50 \mathrm{~cm}$. This makes the use of simpler and therefore cheaper cables possible. Based on signal integrity simulations we chose compact flat cables from Samtec with a width of up to $10 \mathrm{~cm}$ and a capacity of up to 80 pairs of differential signals, i.e., up to 10 links can be routed via one single cable. In Fig. 4b we show the results of a so-called eye diagram measurement. Such diagrams

\footnotetext{
${ }^{3}$ We operate the links at $2.5 \mathrm{GHz}$ instead of $3.125 \mathrm{GHz}$ used in standard $10 \mathrm{GbE}$.
} 
show the overlay of a large number of snapshots sampled with respect to a reference clock. Only if the signal is stable within a certain time window and if transitions between logical ' 0 ' and ' 1 ' occur outside this window the data will be sampled correctly. A transition inside the window (i.e., inside the "eye") is likely to result in a bit error.

The QPACE node cards are interconnected as a 3-dimensional torus. The largest partition which fits on a single backplane is of size $(x, y, z)=(1,4,8)$. To increase the number of nodes in the $y$-direction cables connecting backplanes in the vertical direction have to be used. Similarly, in the $x$-direction backplanes are connected in the horizontal direction. In order to have some flexibility in re-partitioning the machine we make use of a feature of the network PHYs used on the node cards, which come with two serial interfaces. Controlled by software it is possible to select either the primary or the redundant interface. By connecting some of these redundant interfaces to additional backplane lines it is possible to partition the machine in different ways. In Fig. 5 we show how this can be done for a set of 8 nodes connected in the $z$-direction. Given this flexibility we can partition the machine such that we have

1. $1,2,4,8$ nodes in the $z$-direction.

2. $1,2,4,8$ or 16 nodes in the $y$-direction.

3. 1,2 or $2 N$ nodes in the $x$-direction, where $N=1,2, \ldots$ is the number of interconnected racks. A change of $N$ requires re-cabling.

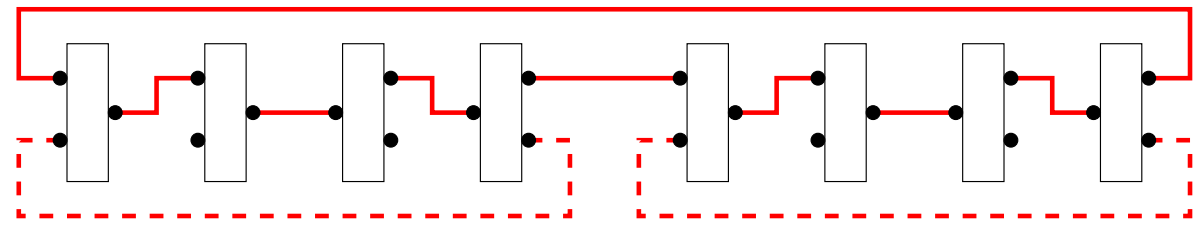

Figure 5: The picture shows 8 node cards which by switching between PHY interfaces in 1 dimension (here: $z$-direction) can be either connected as 1 ring of 8 or 2 rings of 4 node cards.

To measure the performance of our network we use the following benchmarks:

- A ping-pong type of test was implemented to estimate the latency. Here node $A$ sends a message of size 128 Bytes (i.e., 1 packet) from its LS to node $B$. Once the data have arrived in the destination LS of node $B$ this node returns the packet back to $A$. We estimate the latency by measuring the round-trip time on node $A$ and divide this number by 2 .

- An exchange type of test was implemented to measure the bandwidth. In this test node $A$ sends a message to $B$ and vice versa. We allow for multiple messages to be sent concurrently.

With this setup we found the latency to be about $3 \mu$ secs. We also measured the time needed starting from the point where the packet arrives at the transmit FIFO until the DMA engine of the receiver link moves the packet to the processor. Here we measured a latency of about $0.5 \mu$ secs. A large fraction of this latency is due to the logic in the PHYs (where data encoding and decoding 
has to be performed). But the overall latency is largely dominated by the time needed to move data from the processor to the NWP and vice versa.

The bandwidth depends on the message size and the number of concurrent communications. We show the results from different measurements in Fig. 6. As can be seen from this picture it is possible to get close to the theoretical maximum bandwidth of 0.9 GBytes/sec.

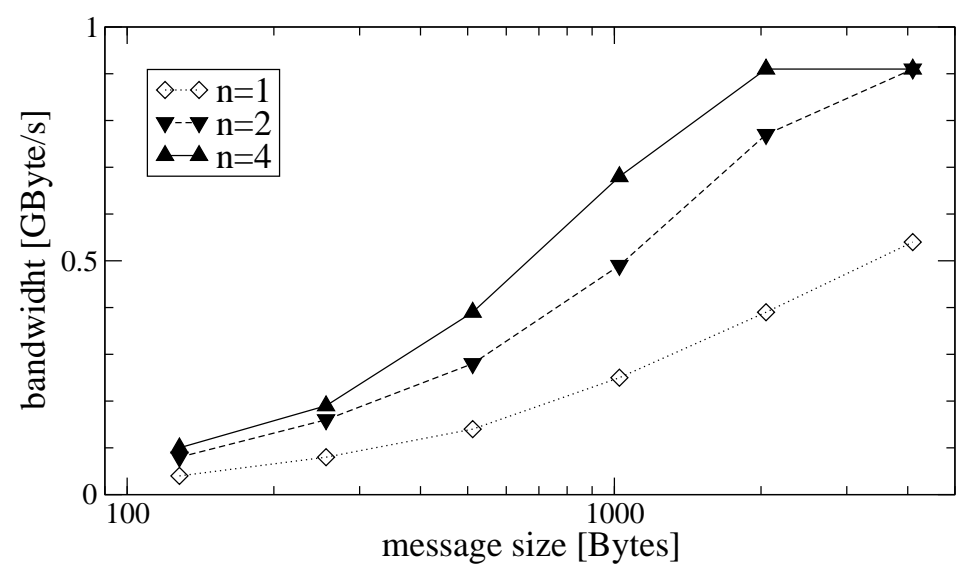

Figure 6: Bandwidth measured on a single torus network link as a function of the message size, where $n$ is the number of messages which are in flight concurrently.

\section{Cooling and power efficiency}

One of the biggest challenges for the integration of the node cards into a single system was the cooling. For all currently operated supercomputers air is used to remove the heat from heat-sinks (or heat-pipes) connected to the electronic components which generate the heat, e.g., the processor. Sometimes liquid cooling is used for heat exchangers inside the rack to cool the air before it either re-enters the computer or leaves the rack. Although this setup is widespread it has its limitations. Air is per se not very suitable for heat transport. As a result large amounts of air have to be pushed through the system, which requires a large amount of space and fans. Increasing the space between the node cards has impact on the costs, because a larger number of backplanes and racks is needed as well as longer and therefore higher quality cables have to be selected. The required fans have an impact on operation by increasing operational and maintenance costs due to increased power consumption and the risk of failure.

On the other hand, liquid cooling is often considered to be risky because it brings water close to expensive components that will be damaged when exposed to water. Liquid cooling solutions tend to be expensive and often have significant impact on maintenance as electronic components may become difficult to access.

The challenge for QPACE was to realize a completely new liquid cooling concept which would address the typical disadvantages of liquid cooling. The node cards are packed into thermal boxes made of aluminium. These boxes act as a single big heat sink conducting the heat from the internal components to the surface. The thermal box is then connected to a cold plate. Water is pumped 
through channels inside the cold plate which moves the heat out of the system. See Fig. 7a for details.

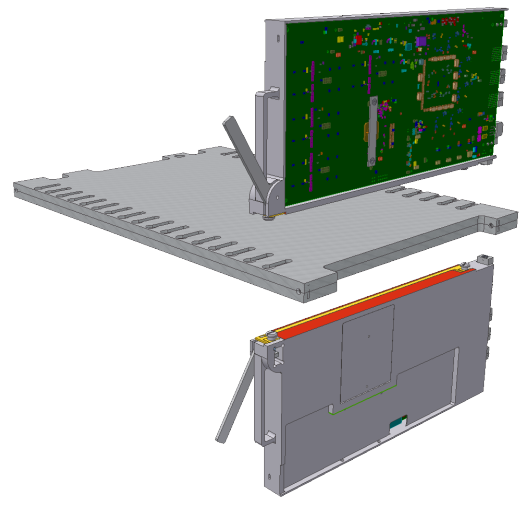

(a) Node cards with coldplate

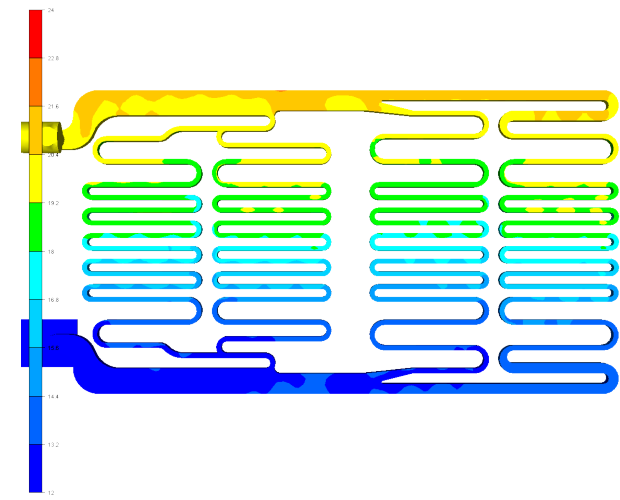

(b) Temperature distribution in cold plate

Figure 7: The left panel shows a cold plate together with node cards, one about to be attached from above, the other from below. The red strip indicates the thermal interface between node card and cold plate. Once the node cards are connected to the cold plate the handle attached to the node card has to be moved into closed position. Springs then press the node card onto the cold plate. In the right panel the result of a simulation of the temperature distribution inside the water-conducting channels of the cold plate are shown.

There is no water flowing through the thermal box and a so-called dry connection is used to connect the node card with the cooling system. Therefore, after installation of the machine the water circuit does not have to be opened for almost any of the expected maintenance operations. This eliminates the need for expensive, self-healing valves often used in other systems.

There are two critical thermal interfaces which have to be kept under control. The first interface is between the electronic components on the node card and the thermal box. From inside, the thermal box had to be carefully designed according to the height of the components and the amount of heat they generate. Thermal grease is used to establish a good thermal contact between these components and the aluminum. Particular demanding is the design near the processor which generates $>50 \%$ of the heat on the board. The other components which need cooling are the memories, the FPGA, the network PHYs, and the power converters.

The second important thermal interface is between the thermal box and the cold plate. The heat has to be conducted through a rather small surface of about $40 \mathrm{~cm}^{2}$. In order to avoid thermal grease, which would be rather inconvenient during maintenance, a suitable type of silicon oil has been selected to improve the thermal contact. Using oil, which generates only a rather thin film, mandated an extremely flat surface on both sides of the interface. Springs mounted in the thermal box additionally improve contact by pressing the node card onto the cold plate after the node card has been mounted.

The cold plate has to be carefully designed in order to make sure that all 16 node cards mounted from the top side and the 16 node cards mounted from below are equally well cooled. In Fig. $7 \mathrm{~b}$ we show the results for the temperature distribution within the cold plate from a simulation. In 
this picture the water enters the cold plate at the lower left corner from where it is distributed over the whole length of the cold plate via a channel with a relatively large diameter. When the water meanders through the small channels it passes from one end of the node cards to the other end, slowly heating up. The warm water is collected in another broader channel and leaves the cold plate at the upper-left corner of the cold plate. It is important to notice that there is only a temperature gradient from bottom to top, from left to right the temperature is constant, i.e., all node cards are cooled equally well. The results of this thermal simulation have been confirmed by temperature measurements using the final system.

An important feature of the QPACE cooling system is the small temperature difference $\Delta T$ between the temperature of the water inside the cold plate and the temperature at the processor. This fact indicates that the thermal interfaces described above are well under control. Even at maximum load we measure $\Delta T \lesssim 30-40^{\circ} \mathrm{C}$. Taking into account that operation of the processor is specified for temperatures up to $95{ }^{\circ} \mathrm{C}^{4}$ using an inlet water temperature $>30{ }^{\circ} \mathrm{C}$ is a feasible option. During test runs we have successfully operated completely populated backplanes with 32 node cards with a water inlet temperature of up to $35^{\circ} \mathrm{C}$ and all node cards running a synthetic benchmark (called PowerLoad SPU) which maximizes the node cards' power consumption.

At high load the temperature at the outlet is about $5{ }^{\circ} \mathrm{C}$ warmer than at the inlet. With an inlet water temperature $>40{ }^{\circ} \mathrm{C}$ the system is able to generate hot water in a temperature range which is at the lower limit to be used for heating and to make free air cooling possible year-round. Using thermal grease for the thermal interface between thermal box and cold plate it is in principle possible to reach higher temperatures.

The cold plates are part of a closed water circuit with a cooling station CoolTrans from Knürr. This station regulates the temperature of the cooling water taking also humidity into account. The latter is necessary to ensure that the temperature in the water circuit stays above the dew point.

While the cooling system is powerful and was designed with sufficient margins, reduction of power consumption continued to be a major target of the QPACE project. The PowerXCell 8i processor is already very efficient in terms of power consumption per floating-point processing power. This statement is supported by the dominance of the PowerXCell 8i-based systems in the top positions of the Green500 list [10].

For the QPACE node cards a further reduction of power consumption could be achieved by tuning the processor voltage for each node card individually. Using the already mentioned synthetic benchmark PowerLoad SPU we measured on a set of 32 node cards from the production batch a reduction by $12 \%$. For this benchmark the average power consumption is about $115 \mathrm{~W}$ per node card. Real applications will not reach the same density of operations as a synthetic benchmark. We therefore expect the average power consumption per node card to be $O(100) \mathrm{W}$.

\section{Operating system, I/O network and front-end integration}

Once the service processor has powered on the PowerXCell 8i, the processor starts executing the firmware, which provides a glue layer between hardware and the operating system. Like

\footnotetext{
${ }^{4}$ The processor can even sustain higher temperatures without being damaged, but with no guarantee for correct functional behavior.
} 
for other PowerXCell 8i-based systems the Slimline Open Firmware (SLOF) is used [11]. The firmware boots the node card into Linux. We are using a standard Fedora distribution with a more recent, slightly modified kernel. The QPACE proprietary devices required the addition of a few additional kernel modules (some of them have become part of the standard Linux kernel). This includes drivers for Ethernet, UART, torus network and global signals.

The Linux operating system is loaded via the Gigabit Ethernet network. With the data link layer as implemented on the QPACE node card it is possible to saturate the bandwidth provided by the underlying physical link layer. Evaluations performed within a QPACE rack have shown a node-to-node ping round-trip time of $50 \mu \mathrm{sec}$ and a sustained TCP bandwidth of 0.1237 GBytes/sec in jumbo frame mode. In this mode the theoretical maximum bandwidth of Gigabit Ethernet is 0.1239 GBytes/sec. Results from these benchmark measurements are shown in Fig. 8. Note that this bandwidth will not be achieved once a larger number of nodes is involved due to limited capabilities of the Ethernet switches.

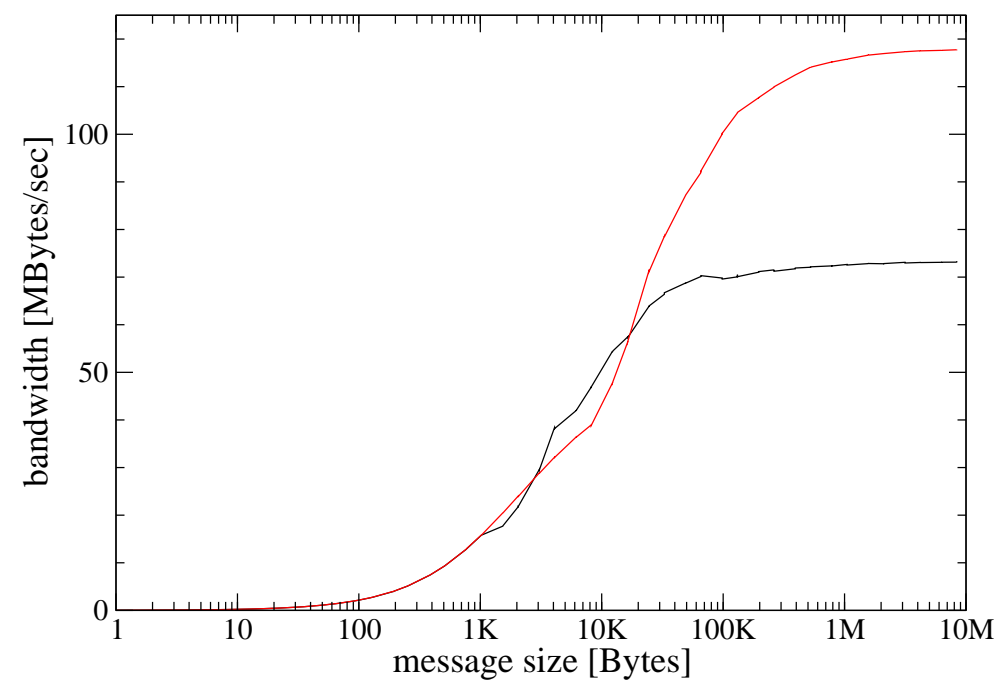

Figure 8: Ethernet bandwidth in units of MBytes/sec measured on a link connecting 2 node cards as a function of the message length. The red curve has been obtained using jumbo frames.

All node cards within one rack are connected to 6 first-level switches. Per rack there are 24 Gigabit Ethernet uplinks connecting the first-level to the second-level switches, which are part of the front-end system. An external bandwidth of $O(2-3)$ GBytes/sec per rack is sufficient for non-I/O intensive lattice QCD applications, like the generation of gauge fields with dynamical fermions. For more bandwidth it would be necessary to upgrade the switches (e.g., by adding 10-GbE uplinks).

The front-end systems of the currently deployed QPACE installations consist of 8 IBM System $x$ Linux servers. A login server acts as central user access point where users can login to submit jobs and manage their data. The so-called master server acts as central system control and monitoring instance. All other servers will be used to provide fast disk storage access. The number of disks attached to these servers is sufficient to sustain a bandwidth of up to 2 GBytes/sec.

We plan to deploy a Lustre parallel file system as soon as (in the near future) clients will become available for the Linux kernel currently used for QPACE. 


\section{Application code and performance}

The first and main challenge of porting application code to QPACE is related to the PowerXCell $8 \mathrm{i}$ architecture, which is quite different from commonly used x86 and PowerPC processors causing an obvious lack of portability. The difficult part is the implementation of the application on the Synergistic Processing Elements (SPE) ${ }^{5}$ The programmer herself has to take care of data management, i.e., allocation of Local Store (LS) space and explicit DMA get and put operations to load data from or store data to main memory.

For optimizing code on QPACE one faces the following challenges:

- With essentially all lattice QCD kernels being memory bandwidth limited it is mandatory to reduce the number of memory accesses to an absolute minimum and to optimize re-use of data in the on-chip LS.

- When parallelizing the application communication has to be organized in such a way that the network latencies of $O(10,000)$ clock cycles are hidden.

- Efficient use of the SPU's floating-point pipeline, i.e., SIMDization of the code.

When implementing a solver, where performance is completely dominated by the performance of the fermion matrix times vector multiplications, an obvious strategy is to choose a data layout which allows for a processing of single blocks. One such strategy is described in [6]. A more natural approach is based on the Domain Decomposition algorithm [12]. This algorithm has the advantage that a very high performance can be achieved at reasonably large local lattice size. A sustained performance of $O(45) \%$ has been measured for the internal block solver. Mainly due to the required memory access the overall solver performance drops to $O(20) \%$ (with room for further improvement).

Some groups which reported on porting lattice QCD kernels or full lattice QCD applications to the Cell processor either avoided some optimization problems, e.g., by restricting themselves to the on-chip memory [13], or have limited their efforts to optimize their data layout [14]. An analysis of different optimization strategies can be found in [15].

The main purpose of QPACE is to provide the compute power needed to generate gauge configurations with dynamical fermions. We are currently working on application programs using Clover fermions and domain wall fermions. But there are also attempts to port, e.g., multi-grid methods [16] to QPACE. Furthermore, a 2D code for multi-phase fluid dynamics based on the Lattice Boltzmann approach has been written and validated on a 32 node QPACE system, and a (DP) efficiency of $\simeq 20 \%$ of peak has been reached. Further performance optimizations are possible. A preliminary report of this activity is in[17].

\section{Project organization}

Within the QPACE project a new model of collaboration that was new to all partners has been developed. In the QPACE setup we have on the one hand a set of academic partners and on the

\footnotetext{
${ }^{5}$ It is easy to port applications to the Power Processing Element (PPE), but high performance can only be reached when using the SPEs.
} 


\begin{tabular}{|l|l|}
\hline Peak performance single/double precision & $26 / 52$ TFlops \\
\hline Rack size $(\mathrm{w} \times \mathrm{d} \times \mathrm{h})$ & $80 \times 120 \times 250 \mathrm{~cm}^{3}$ \\
\hline Weight & $O(1,000) \mathrm{kg}$ \\
\hline Maximum power consumption & $<35 \mathrm{kWatts}$ \\
\hline Average power consumption & $O(29) \mathrm{kWatts}$ \\
\hline
\end{tabular}

Table 2: Key parameters of a QPACE rack.

other hand one strong industrial partner, IBM. Some of the academic institutions as well as IBM had a double role of being partner and customer/vendor at the same time.

While the customer/vendor model was particularly relevant during the manufacturing process in order to define clear responsibilities, the partnership model has important advantages for the development process. A joint development team was able to quickly adjust and refine the requirements and to bring together the know-how and skills to react quickly and flexibly to all the problems which needed to be addressed and solved.

The project has been carried out in an extremely short period of time. Officially the project started in January 2008. At this point a number of major design decisions had already been made and first experiments addressing key technological challenges had been performed. Already in June 2008 the first versions of the node card became available, prototypes of backplane and root card followed in July 2008. In December 2008 and January 2009 extensive hardware integration tests have been performed, which where mandatory to decide on release for manufacturing. All components where finally released for manufacturing in February and March 2009. In April 2009 the first components started to arrive from the manufacturers and the machine integration phase started. After pre-integration of the racks at the University of Regensburg, the racks were shipped to the IBM Böblingen lab. There all racks were first fully assembled and tested before being deployed at the installation sites. Deployment both at the Jülich Supercomputing Centre and the University of Wuppertal has been completed in August 2009 and a test operation phase has started.

\section{Conclusions and outlook}

QPACE is a new, scalable parallel computer based on the PowerXCell 8i processor which is optimized for lattice QCD applications. While a rack consists mainly of custom designed components, the compute power is provided by commodity processors which are interconnected by a custom network. Key parameters of a QPACE rack are collected in Table 2.

Within this project we tried to explore new technical concepts. The design highlights of the QPACE architecture include:

- An I/O fabric has been implemented on an FPGA which is directly attached to the PowerXCell 8 i processor.

- The processors are interconnected by a fast lattice QCD optimized, low latency torus network. 
- A custom system design makes it possible to mount 256 node cards within a single rack, thus providing a very high compute density.

- The compactness of the design is facilitated by a novel, cost-efficient liquid cooling system.

- The power consumption of the node cards has been further reduced in the course of this project making QPACE one of the most power-efficient currently available parallel computers.

The QPACE hardware has been deployed at two installation sites, the Jülich Supercomputing Centre and the University of Wuppertal. The installations consist of 4 racks each, i.e., an aggregate peak performance of 200/400 TFlops (double/single precision).

During burn-in of the hardware in the integration phase a few defective components had been found. After deployment at the installation sites and since start of the test operation phase almost no defects occurred.

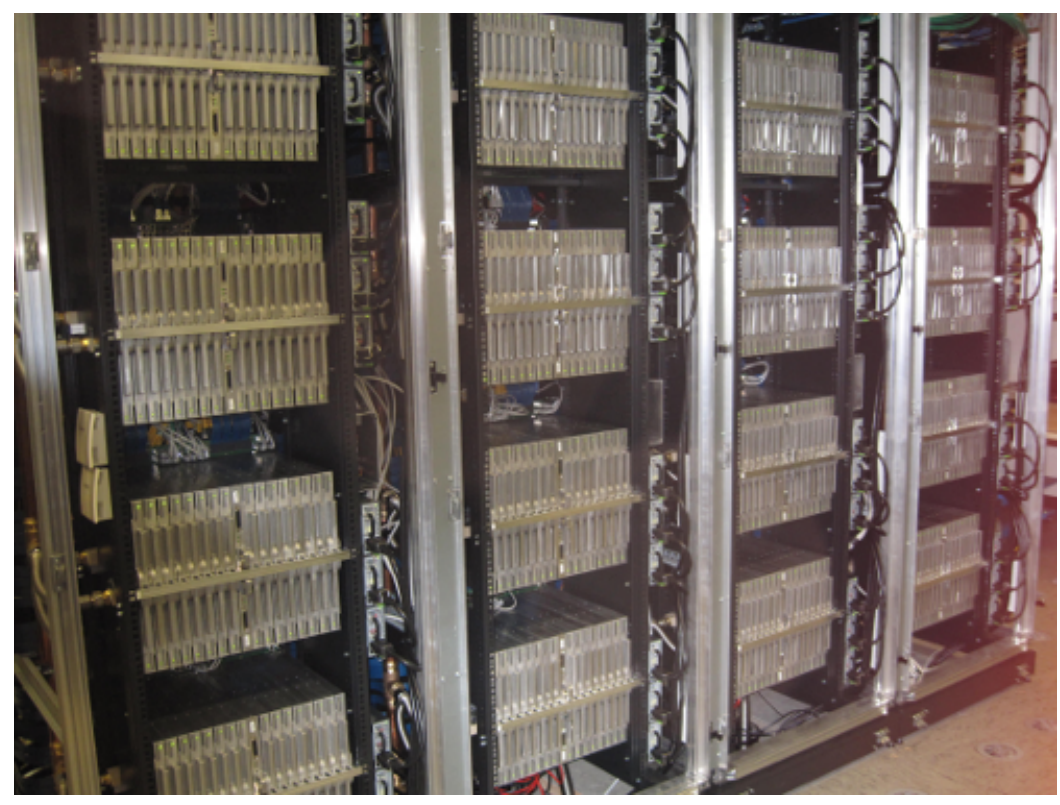

Figure 9: 4 QPACE racks installed at the Jülich Supercomputing Centre.

While there are still some ongoing development activities, as of today no serious flaws have been identified which could be attributed to wrong design decisions. The technical concepts behind the QPACE architecture have essentially been proven to be successful. One example is the use of FPGAs for implementing a custom network to interconnect commodity processors. However, this particular example also shows that there are non-trivial technical challenges which have to be solved. With increasing resource usage it becomes more and more difficult to fit the design into the FPGA. This area still requires further attention.

Currently application codes are being ported to the new architecture. For a key application kernel, a solver for Clover-fermions based on the Domain Decomposition algorithm, a sustained 
performance of $O(20) \%$ has been reached. As there is room for improving this performance to up to $O(30) \%$ we hope for a (single-precision) sustained performance of 10-16 TFlops per rack.

We plan to reach production mode by end of 2009/beginning of 2010. This would put us in a position to present physics results generated on QPACE at the next Lattice Symposium.

\section{Acknowledgements}

We acknowledge the funding of the QPACE project provided by the Deutsche Forschungsgemeinschaft (DFG) in the framework of SFB/TR-55 and by IBM. We furthermore thank the following companies who contributed significantly to the project in financial and/or technical terms: Axe Motors (Italy), Eurotech (Italy), Knürr (Germany), Xilinx (USA) and Zollner (Germany).

\section{References}

[1] F. Belletti et al., Computing for LQCD: apeNEXT, Computing in Science \& Engineering 8 (2006) 18.

[2] P.A. Boyle et al., Overview of the QCDSP and QCDOC computers, IBM J. Res. \& Dev. 49 (2005) 351.

[3] IBM, Cell Broadband Engine Architecture, Version 1.0, 8 August 2005.

[4] K.J. Barker et al., Entering the petaflop era: the architecture and performance of Roadrunner, Proc. of the 2008 ACM/IEEE conference on Supercomputing .

[5] http://www.top500.org/list/2009/06/100 (accessed on 13 October 2009).

[6] F. Belletti et al., QCD on the Cell Broadband Engine, PoS (LAT2007) 039 [arXiv:0710.2442 [hep-lat] ].

[7] G. Goldrian et al. QPACE: Quantum Chromodynamics Parallel Computing on the Cell Broadband Engine, Computing in Science \& Engineering, Vol. 10, Issue 6 (2008).

[8] I. Koutsou, A. Nobile, H. Simma, in preparation.

[9] I. Ouda, K. Schleupen, Application Note: FPGA to IBM Power Processor Interface Setup, IBM Research Report, RC24596 (W0807-021) July 2, 2008.

[10] http://www.green500.org/lists/2009/06/list.php (accessed on 17 October 2009).

[11] H. Schick et al., LAB: Slimline Open Firmware, http://www.power.org/devcon/07/Session_Downloads/PADC07_Schick_Slimline_Open_Firmware.pdf (accessed on 17 October 2009).

[12] M. Lüscher, Lattice QCD and the Schwarz alternating procedure, JHEP 0305, 052 (2003) [arXiv:hep-lat/0304007].

[13] J. Spray, J. Hill and A. Trew, Performance of a Lattice Quantum Chromodynamics Kernel on the Cell Processor, Comput. Phys. Commun. 179 (2008) 642 [arXiv:0804.3654 [hep-lat] ].

[14] G. Shi, V. Kindratenko and S. Gottlieb, Cell processor implementation of a MILC lattice QCD application, POS (LAT2008)026 [arXiv:0910.0262 [hep-lat]].

[15] K.Z. Ibrahim and F. Bodin, Efficient SIMDization and data management of the Lattice QCD computation on the Cell Broadband Engine, Scientific Programming, Volume 17, Issue 1-2 (January 2009). 
[16] D. Ritter et al., http://www10.informatik.uni-erlangen.de/Publications/Talks/2009/Ritter_Copper_09.pdf (accessed on 17 October 2009).

[17] F. Belletti et al., Multiphase lattice Boltzmann on the Cell Broadband Engine, Il Nuovo Cimento della Societa' Italiana di Fisica, 2009. 